\title{
Entropies Galore!
}

\author{
P. T. Landsberg \\ Faculty of Mathematical Studies \\ University of Southampton \\ Southampton, SO17 1BJ, UK. \\ Received 07 December, 1998
}

\begin{abstract}
The functional properties of the entropy gives rise to 6 possible types of thermodynamics. Additivity or superadditivity or subadditivity are closely related to extensivity and this is one of the characteristics used to distinguish the 6 types. We give examples (some are somewhat academic) of all of these, except one. For this purpose we draw also on black hole systems which have been proposed. Some of these systems have subadditive entropies, i.e. they tend to fragment rather than clump. After proposing a new entropy function we raise the problem of how to select from these entropy functions. Are some better than others?
\end{abstract}

\section{Introduction: Meaning of ex- tensivity}

Discussions of extensivity in thermodynamics go back to the 1960ies [1]. There are several non-equivalent definitions of the extensivity of functions. They are $[2,3]$, if $x, y$ are three-dimensional vectors $\mathrm{x}=\left(x_{1}, x_{2}, x_{3}\right)$,

$$
\begin{aligned}
& f(\mathbf{x}+\mathbf{y})=f(\mathbf{x})+f(\mathbf{y}) \\
& f(2 \mathbf{x})=2 f(\mathbf{x}) \\
& f(\lambda \mathbf{x})=\lambda f(\mathbf{x}) \quad(\lambda>0)
\end{aligned}
$$

A statistical mechanical definition can be added. It utilises ratios $\rho_{1} \equiv x_{1} / x_{3}, \rho_{2} \equiv x_{2} / x_{3}$ and states that

$$
x_{3}^{-1} f(\mathbf{x}) \sim f\left(\rho_{1}, \rho_{2}\right)
$$

where the limit $x_{1}, x_{2}, x_{3} \rightarrow \infty$ (for $\rho_{1}, \rho_{2}$ fixed) is considered.

These definitions are not equivalent. For example,

$$
f(x)=x_{1}^{2} /\left(x_{1}^{2}+x_{2}^{2}\right)^{\frac{1}{2}}
$$

satisfies (3), but not (1). However, if the assumption

$$
P: \mathbf{x} \text { and } \mathbf{y} \text { are parallel vectors }
$$

is added, then, indeed

$$
\text { (3) }+P \Rightarrow(1) \text {. }
$$

Again, although (1) implies (2), (1) implies (3) only if the assumption

$C_{0}$ : the function $f$ is continuous in its arguments

is added. Without it one can by addition not get much beyond

$$
n f(\mathbf{x})=f(n \mathbf{x}) \quad \text { or } \quad m n^{-1} f(\mathbf{x})=f\left(m n^{-1} \mathbf{x}\right)
$$

where $m$ and $n$ are positive integers. But with $C_{0}$ one can reach (3) from (1) via (6).

In this way a table of implications can be set up for the four definitions of extensivity. It is then found that for larger systems (4) implies the other three definitions. If $C_{0}$ is also assumed, then (1) can join (4) in implying the other three definitions.

Let us adopt (1) therefore as the definition of extensivity in this paper. This is in any case a usual definition. A failure of (1) leads then to superadditivity if the left-hand side is greater than the right-hand side, and to subadditivity if it is smaller.

\section{Types of thermodynamics [4]}

I now recall the possibility of classifying all entropy functions, and hence the associated types of thermodynamics, into eight categories. These are generated by three characteristics each of which may hold $(\mathbf{S}, \mathbf{H}, \mathbf{C})$ or may not hold $(\overline{\mathbf{S}}, \overline{\mathbf{H}}, \overline{\mathbf{C}})$ (see Table I). 
Table I: Types of Thermodynamics
1. $\mathbf{S H C} 2 . \mathbf{S} \overline{\mathbf{H}} C$
3. $\mathrm{SH} \overline{\mathrm{C}}$
4. $\mathbf{S} \overline{\mathbf{H}} \overline{\mathrm{C}}$
5. $\overline{\mathrm{S}} \mathrm{HC}$
6. $\overline{\mathrm{S}} \overline{\mathrm{H}} \mathrm{C}$
7. $\overline{\mathrm{S}} \mathrm{HC}$
8. $\overline{\mathrm{S}} \overline{\mathrm{H}} \overline{\mathrm{C}}$

Here we have

$\begin{array}{llll}\text { Superadditivity } & \mathbf{S :} & S(\mathbf{x}+\mathbf{y}) \geq S(\mathbf{x})+S(\mathbf{y}) & {[c f(1)]} \\ \text { Homogeneity } & \mathbf{H}: & S(\lambda \mathbf{x})=\lambda S(\mathbf{x}) & {[c f(3)]} \\ \text { Concavity } & \mathbf{C}: & S(\lambda \mathbf{x}+(1-\lambda) \mathbf{y}) \geq \lambda S(\mathbf{x})+(1-\lambda) S(\mathbf{y})\end{array}$

Note that $\mathbf{C}$ is quite different from $C_{0}$, introduced above. Also note that here $\mathbf{x}$ and $\mathbf{y}$ can stand for a set of variables or for just one variable.

\section{Types of systems considered}

The first type is inspired by the ideal gas and is specified by the entropy

$$
S=k \ell n\left(a U v^{g} / N^{h}\right)
$$

where $k, a, g, h$ are positive constants and $v$ is the volume, $U$ the internal energy and $N$ the number of particles in the gas. It turns out that this example can generate three types of thermodynamics [4, paper II]:

$h=g+1:$ type $1 . ; H<g+1:$ type $4 . ; h>g+1:$ type 6 .

Of course type 1 is the normal thermodynamics; type 4 reveals the purpose of the example:

For it was given as a simple classical analogue of the thermodynamics of the Schwarzschild black hole, in that it violates $\mathbf{C}$ but keeps $\mathbf{S}$.

Second type: For a Schwarzschild black hole of mass $M, M_{0}$ being a constant, one knows that

$$
S=k\left(M / M_{0}\right)^{2},
$$

Its thermodynamics is of type 4 as well. Indeed one can show that this holds more generally [5] for

$$
S=a m^{\beta}+b .
$$

where $\alpha>0, \beta>1$ and $b$ are positive constants, provided $b$ is small enough (See [5], line 1 of Table II).

The thermodynamics of type 8 , for which $\mathbf{S}, \mathbf{C}$ and $\mathbf{H}$ all fail, is rather rare. But it is found for special case of (10), for example if $(a, b>0)$

$$
\begin{aligned}
& S=a m^{2}+b, \\
& S=\frac{a}{m}+b, \\
& S=-a \sqrt{m}+b,
\end{aligned}
$$

as for certain dilatonic black holes [5].

As third type, consider a $(1+1)$-dimensional black hole, as discussed in [6], for which

$$
S=k \ell n\left(M / M_{0}\right)
$$

In this case one finds type 2 if $M / M_{0}<4$ and type 6 if $M / M_{0}>4$.

In the second and third types there has in effect been only one independent variable which of course simplifies the analysis. Fig. 1 gives a summary of the results in the form of a Venn diagram.

It is seen from Fig. 1 that no example has been given of a thermodynamics of types 3,5 and 7 . Types 3 and 5 are actually logically impossible because

$$
\mathbf{S}+\mathbf{H} \rightarrow \mathbf{C} \quad \text { and } \quad \mathbf{C}+\mathbf{H} \rightarrow \mathbf{S} .
$$

For functions which depend on one independent variable only we know also ([5]) that $\mathbf{H} \rightarrow \mathbf{S}, \mathbf{C}$ which rules out thermodynamics of type 7 . We still need an example of this type. It must involve several independent variables.

Broadly speaking, if $\mathbf{S}$ holds, then two identical systems will tend to "clump" together if they are given a chance, for this way the entropy goes up. This holds for a thermodynamics of types 1,2 and 4 . However if $\mathbf{S}$ fails, as it does in thermodynamics of types 6,7 and 8 , then a system increases its entropy by splitting up into separate systems. This is "fragmentation" [6]. 


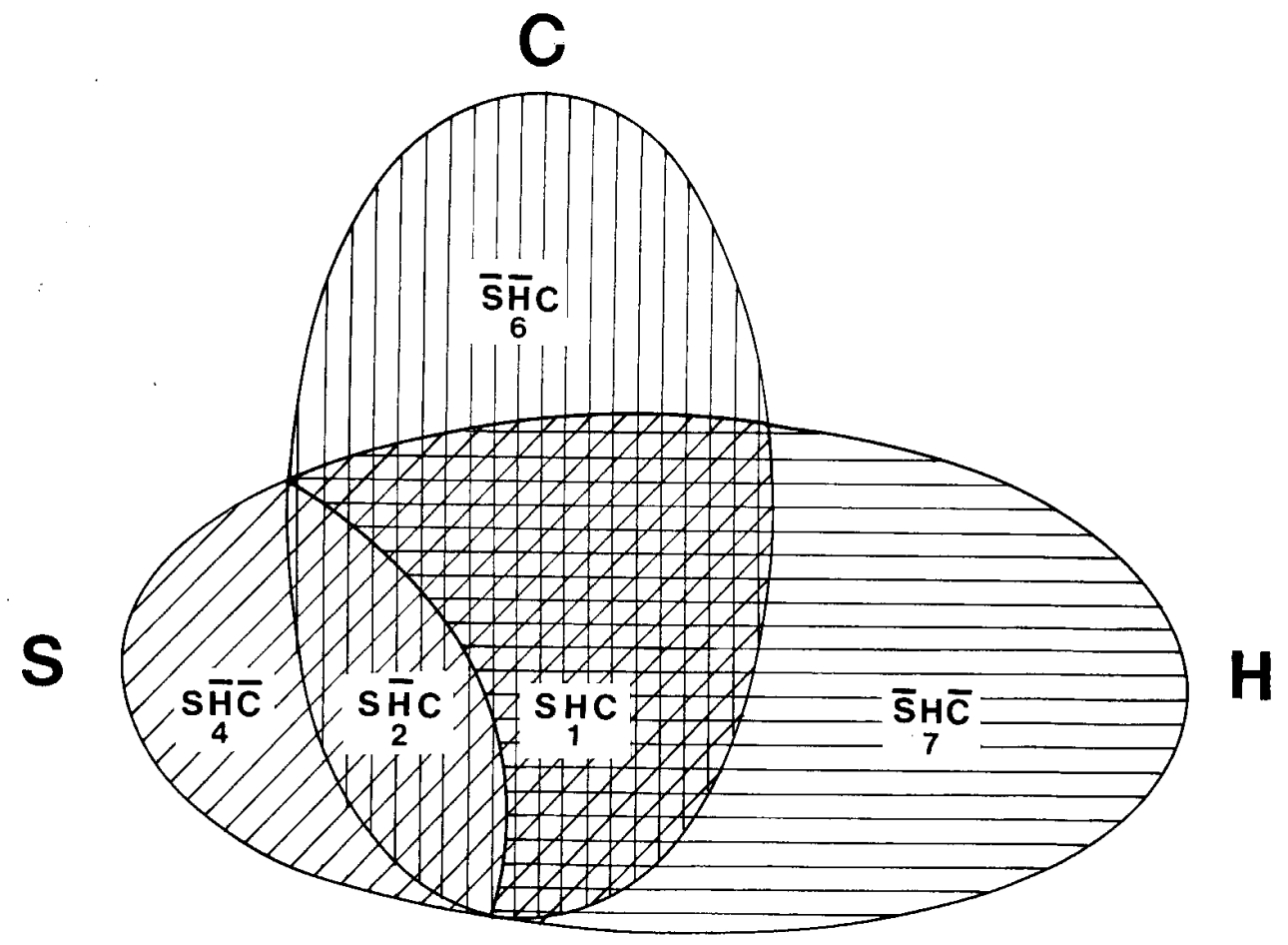

Figure 1. Venn diagram for thermodynamic types.

\section{Entropy choices}

The non-extensive nature of the entropy can be encapsulated in a function $Y$ which vanishes in the case of an extensive entropy:

$$
S(A+B)-S(A)-S(B)=Y S(A) S(B)
$$

where $A$ and $B$ represent the part systems under consideration [7]. Let us add superfixes $R, S, T, U$ for Renyi, Shannon, Tsallis and a new entropy. Let $Q \equiv \Sigma p_{i}^{q}$, where the $p_{i}$ are the probabilities of the various states of the system involved, and let $q$ be a real constant. Then we can construct a table of matching entropies and $Y$-functions [8]:

$$
\begin{aligned}
S^{(R)}(A) & =\frac{k}{1-q} \ln Q & Y^{(R)} & =0 \\
S^{(S)}(A) & =-k \sum_{i} p_{i} \ln p_{i} & Y^{(S)} & =0 \\
S^{(T)}(A) & =\frac{k}{1-q}(Q-1) & Y^{(T)} & =\frac{1-q}{k} \\
S^{(U)}(A) & =\frac{k}{1-q}\left(1-\frac{1}{Q}\right) & Y^{(U)} & =\frac{q-1}{k}
\end{aligned}
$$

For each $S$ one has $\lim _{q \rightarrow 1} S^{(i)}(A)=S^{(S)}(A)$, and the new entropy satisfies

$$
S^{(U)}(A)=S^{(T)}(A) / Q
$$


A great deal of work has been done using the Tsallis expression $S^{(T)}$. The object was to find various fits to experimental data and limits on values of $q$, or, more precisely, on the amount by which $q$ is likely to differ from the Shannon value of unity. In the case of the cosmological background radiation, for example, the best $q$ value lies very close to unity [9]:

$$
|q-1| \sim 10^{-4}-10^{-5}
$$

Physical reasons for departures from simple laws of any kind always exist, since it is well-nigh impossible to take account of all interactions. There is always a further stage! In this particular case one hopes to get a grip on the long-range gravitational effects by going from the Shannon to the Tsallis entropy.

The are, of course, alternatives to (14). Although (15) is a new suggestion [8], people have had the question of uniqueness at the back of their minds even though there must by now be something like 40 applications of the Tsallis entropy. That is why interesting uniqueness theorems have appeared [10].

Still, alternative entropies can be constructed, based on the process that yielded (15) and others [11]. How do we chose among them? Is the Tsallis entropy the only reasonable contender? And if so, why is this so? Is there a general significance of the parameter $q$ ? Some future studies could usefully be devoted to these questions.

\section{References}

[1] L. Galgani and A. Scotti, On subadditivity and convexity properties of thermodynamic functions, Pure and Applied Chemistry 22, 229 (1970). Also in Proc. Intern. Conf. on Thermodynamics (Ed P. T. Landsberg),
Cardiff 1970.

P. T. Landsberg, Main ideas in the axiomatics of thermodynamics,

Pure and Applied Chemistry 22, 215 (1970).

[2] J. Dunning-Davies, On the meaning of extensivity, Phys. Lett. 94A, 346 (1983). Extensivity and the Gibbs-Duhem equation, Phys. Lett. 37A, 327 (1983).

[3] J. Dunning-Davies and P. T. Landsberg, Logical relations among different definitions of "extensivity", Phys. Lett. 107A, 383 (1985).

J. Dunning-Davies, Thermodynamics of non-extensive system, J. Phys. Chem. Solids 49, 705 (1988).

[4] P. T. Landsberg and D. Tranah, Thermodynamics of non-extensive entropies I, Collective Phenomena 3, 73 (1980).

D. Tranah and P. T. Landsberg, Thermodynamics of non-extensive entropies II, Collective Phenomena 3, 81 (1980).

[5] P. T. Landsberg and R. B. Mann, Thermodynamic Classifications and dilatonic black holes, Gen. Relativity and Gravitation 29, 1269 (1997).

[6] P. T. Landsberg and R. B. Mann, New types of thermodynamics from $(1+1)$-dimensional black holes, Class. Quantum Grav. 10, 2373 (1993).

[7] C. Tsallis, Nonextensive physics: a possible connection between generalized statisical mechanics and quantum groups, Phys. Lett. A 195, 329 (1994).

[8] P. T. Landsberg and V. Vedral, Distributions and channel capacities in generalized statistical, mechanics Phys. Lett. A 247, 211 (1998).

[9] A. B. Pinheiro and I. Roditi, Non extensive thermostatistics and deformed structures, Phys. Lett. A 242, 296 (1998).

[10] R. J. V. dos Santos, Generalization of Shannon's theorem for Tsallis entropy, J. Math. Phys. 38, 4104 (1997).

[11] A. R. R. Papa, On one-parameter-dependent generalizations of Boltzmann-Gibbs statistical mechanics, J. Phys. A 31, 5271 (1998). 